\title{
A magneto-inductive wave wireless power transfer device
}

\author{
CHRISTOPHER J. STEVENS
}

\begin{abstract}
Magneto-inductive waves are a form of propagation which only exists in certain types of magnetic metamaterials formed from inductively coupled resonant circuits. We present an investigation of their potential as contactless power transfer devices capable of carrying power along a surface between suitably prepared terminals while simultaneously offering a broadband data channel. Input impedances and their matching conditions are explored with a view to offering a simple power system design. A device with $75 \%$ peak and $40 \%$ minimum efficiency is demonstrated and designs with potential for better than $70 \%$ mean and $90 \%$ peak are reported. The product of planar magnetic coupling and metamaterial cell $Q$ factor is determined to be a key optimization parameter for high efficiency.
\end{abstract}

Keywords: Metamaterial, Induction, NFC

Received 1 October 2014; Revised 24 February 2015; first published online 24 April 2015

\section{INTRDDUCTION}

Wireless power transfer is an old topic [1] of much current interest $[2,3]$ with significant competition between standards and approaches. Most approaches utilize magnetic induction to carry power from a transmitting coil to one or more receiving units. Operation frequencies between $100 \mathrm{kHz}$ and tens of $\mathrm{MHz}$ have been considered with the license-free ISM bands at 6.78 and $13.56 \mathrm{MHz}$ being attractive choices. Metamaterials have been demonstrated to usefully contribute to these systems by helping to concentrate magnetic flux on its way from transmitter to receiver [4] and have also been considered as power delivery media $[5,6]$. Following the original work on two terminal systems [2], where a power source drives a resonant transmitting antenna delivering power to a load via a single receiving coil, a number of researchers have considered approaches for increasing the range of efficient power transfer. Several methods have been employed to increase the range, notably the use of passive, resonant, relay coils to increase the oscillating magnetic flux at the receiving antenna. There are two main configurations for relays that have been studied - axial [7] and planar [8,9]. Such relay coil arrays are identical to metamaterial structures.

In general, metamaterials are repeating arrays of resonant electromagnetic elements and in this work we are concerned with magnetically active varieties [10]. Periodic arrays of coupled circuits such as those employed in metamaterials generally support several modes of signal propagation. In 2002, magnetic metamaterials were discovered to exhibit a novel type of wave propagation known as a magneto-inductive

Department of Engineering Science, The University of Oxford, Parks Road, Oxford OX1 3PJ, UK. Phone: +44 1865283272

Corresponding author:

C. J. Stevens

Email: chris.stevens@eng.ox.ac.uk wave (MIW) [11] which may only propagate within its host metamaterial.

In the past work, MIWs have been considered as novel waveguides [12], for distributed data transfer systems [10, 13 ] and to carry signals in magnetic resonance imaging [14]. A significant advantage of this type of waves for power transfer is their strongly magnetic character which leads to efficient coupling to resonant electric circuits in their near field. By their very nature MIW with strong coupling offer low loss and wide bandwidth channels [10]. Thus a good power transfer structure is also perfectly prepared to offer a highbandwidth communication channel with data capacity well above traditional near field coupled (NFC) systems.

This paper deals with the application of MIW to power transfer via a one-dimensional $(1 \mathrm{D})$ structure which supports them. When driven by a suitable source and coupled to a power receiver MIW carry energy from source to load wherever it is placed on along the device. As an example, such a device could be used to recharge a number of mobile telephones equipped with contactless power receivers. A simple scheme for this is shown in Fig. 1 along with its equivalent circuit and it is this scheme that we consider for the rest of the paper.

Section II details the modeling of MIW power transfer lines and is divided into three parts.

Section IIA describes the properties of MIWs, the benefits they convey in terms of bandwidth and offers a simple figure of merit for structure design.

Section IIB uses MIW characteristic impedance to determine the input impedances for semi-infinite MIW-based power transfer structures for two different source connections.

Section IIC considers finite length structures and the impact of standing waves on input impedances, finally calculating power transfer efficiency for a model line under three different constraints. 

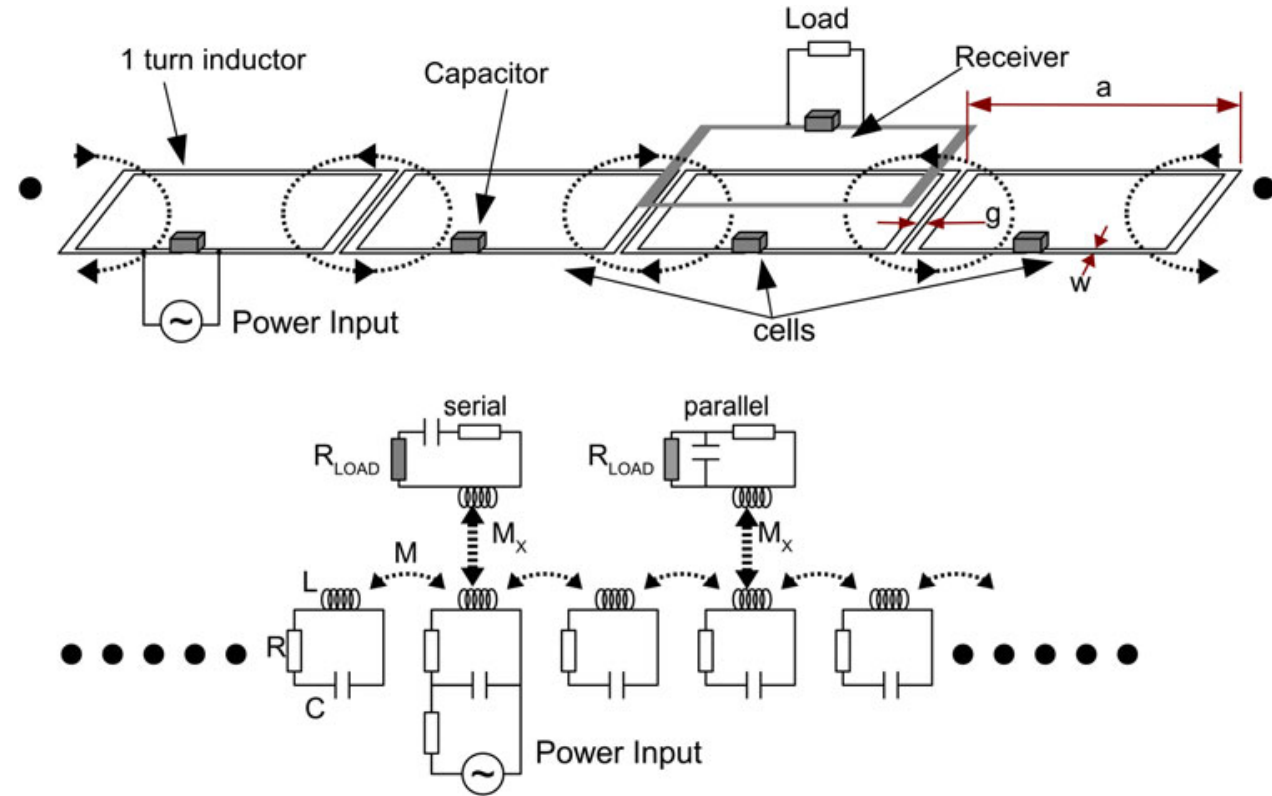

Fig. 1. Simple structure for a MIW-based near-field power transfer structure using a MIW line. Equivalent circuit showing the first neighbor coupling and receiver coupling terms.

Section III reports results of a simple experiment on one such finite line and compares the performance with predicted values.

Section IV considers future approaches to further optimizing the structure in light of the results with potential for power transfer efficiency in excess of $80 \%$ to loads at a range of 800 $\mathrm{mm}$ along a MIW line from the driving source.

\section{MIWS ANDPDWER TRANSFER}

Metamaterials for resonant frequencies are generally formed using periodic arrays of resonant circuits ("cells"). Magnetic metamaterials (being those designed to exhibit a strong magnetic permeability) utilize simple series resonant circuits (inductance $L$, resistance $R$, and capacitance $C$ ), which are often coupled together via the mutual inductance between adjacent cells. Figure 1 shows a typical, $1 \mathrm{D}$ metamaterial structure, whose cells are square tracks with series capacitors bridging a small gap in the conductors forming the LC resonator. The cells are very close to one another so that a current circulating in one cell generates a significant magnetic flux through the neighboring cells and hence they are coupled via a mutual inductance $M$. If a resonant current is induced in one cell, the mutual inductance results in a current being stimulated in the cells neighbors. These in turn stimulate their neighbors and this is the propagation of a MIW [11]. A key feature of these structures is that they are open: the magnetic flux linking the cells extends beyond the structure and provides a region of near field from which nearby structures can draw power or data signals. The power transfer device which is the subject of this paper is formed by connecting an radio frequency (RF) source directly to one of the cells of a conventional MIW guide as shown in the lower pane of Fig. 1. Power is then delivered to one or more receivers in the near field of the guide.

\section{A) MIWs}

Detailed descriptions of the properties of MIWs can be found in the literature $[10,11-15]$ and need not be repeated here. We include the key results below. In general, there is a limited bandwidth available for signal transmission via this type of channel with the bandwidth increasing as the mutual inductance coupling rises while the losses commensurately decrease. In consequence the optimal structure for both power and high-bit-rate data transfer requires that the mutual inductive coupling between cells be as high as possible. This coupling is usually characterized by the parameter $k=2 M / L$, where $M$ is the nearest-neighbor mutual inductance and $L$ is the selfinductance of each cell.

MIWs are described in terms of the currents circulating in each cell (In for the $n$th cell) and the general expression for a MIW in $1 \mathrm{D}$ is $I=I_{\mathrm{o}} e^{(a+j b) n d}$, where $a$ is the attenuation and $b$ is the wave vector, and $d$ is the period of the cells. Attenuation at the cells resonant frequency is then given by equation (1) where $\alpha d$ is the attenuation per cell for MIW transmission (in a structure with a period of $d$ ) and $Q$ is the individual cell's resonant quality factor measured in isolation [16].

$$
\alpha d=\sin h^{-1}\left(\frac{1}{k Q}\right) .
$$

Its very clear that for efficient power transmission in this medium strong coupling (high $k$ ) and low loss (high $Q$ ) resonators are preferred. Hence in optimizing metamaterial power transfer designs, care should be taken that the figure of merit $k Q=2 \omega_{\mathrm{o}} M / R$ be as high as possible. Propagation for waves in such a very low loss system is then governed by a simple dispersion equation.

$$
\cos (\beta d)=\frac{\omega_{\mathrm{o}}^{2}-\omega^{2}}{k \omega^{2}},
$$


where, $\omega_{0}$ is the cell resonant angular frequency $\left(\omega_{0}=1 \sqrt{L C}\right)$. The band limits for MIW transmission are then defined by the frequencies for which $|\cos (b d)|=1$. This provides a simple expression for the bandwidth which is shown in equation (3)

$$
\Delta \omega=\omega_{\mathrm{o}}\left(\frac{1}{\sqrt{1-k}}-\frac{1}{\sqrt{1+k}}\right) .
$$

In a $1 \mathrm{D}$ structure with $|k|=0.3$ the bandwidth available for MIW transmission will be $31 \%$ of the cell resonance frequency $\omega_{\mathrm{o}}[11]$ given by setting $k=0.3$ in equation (3).

\section{B) Input impedance and matching}

For a $1 \mathrm{D}$ MIW there is a simple matching condition [15] when only first-order coupling is significant which states that a structure is perfectly terminated if the last cell in a line includes an extra impedance $Z_{m}$ given by

$$
Z_{m}=j \omega M e^{-(\alpha+j \beta) d} .
$$

In practice, this is a rather difficult situation to achieve as the value of $Z_{m}$ is a complex frequency dependent function and only has a pure real value (i.e., resistive character) at $\omega_{\mathrm{o}}$. Despite this, in terms of determining the match between a source and a MIW line or a load coupled to one $Z_{m}$ allows a simple analysis to be performed.

We have investigated two configurations of source connection directly to one cell of a MIW line, in series with the components of that cell or in parallel across the capacitance of the cell.

We consider two locations for a source directly coupled to the MIW line. First a source coupled to the first or last cell of a line (END). Second, a source coupled to a cell in the middle of the line (MID). Equivalent circuits for the two cases are shown in Fig. 2.

In the END case the first cell of the line, to which the source of internal resistance $R_{s}$ is coupled (in parallel with the resonating capacitor or series with the cell) includes an element of impedance $Z_{m}$. This is sufficient to model the source being connected to the start of a semi-infinite line. For the MID

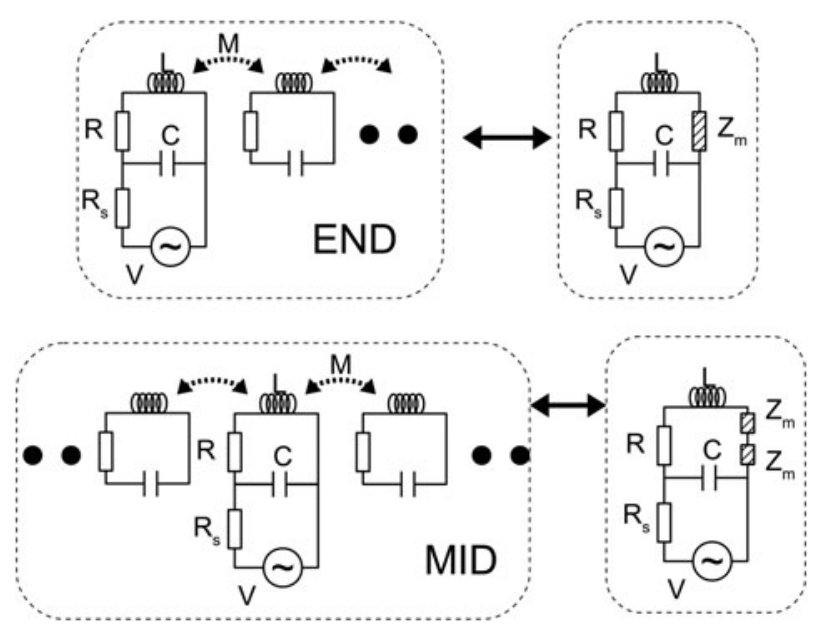

Fig. 2. The two cases for parallel power connection to the MIW line showing the use of the matching impedance $Z_{m}$ to allow calculation for an infinite line. case the driven cell is linked to two semi-infinite lines and so is modeled with two identical impedances $Z_{m}$ for the MIW launched by this source along the two directions of the infinite line. Using these two simplifications the matching condition for any driving frequency can be obtained. Using simple circuit methods the effective input impedances $\left(Z_{\text {in }}=R_{\text {in }}+j X_{\text {in }}\right)$ presented to the source in each case can be derived for these parallel configurations (source connected across input cell's capacitor) as

$$
\begin{aligned}
& \frac{1}{Z_{\text {in }}}=j \omega C+\frac{1}{R+j \omega L+Z_{t}} \quad \text { Parallel, } \\
& Z_{\text {in }}=R+j\left(\omega L-\frac{1}{\omega C}\right)+Z_{t} \quad \text { Series, }
\end{aligned}
$$

where $Z_{t}$ is given by $Z_{m}$ for the END case and $2 Z_{m}$ for the MID case.

Figure 3 shows the results for the two configurations (parallel and series) for both locations (END and MID). Values used in this calculation were derived from FastHenry [17] simulations of $w=1 \mathrm{~mm}$ wide tracks forming square single turn inductors with side length of $a=40 \mathrm{~mm}$, separated by gaps of $g=0.2 \mathrm{~mm}$. Cell resistance is taken to be $88 \mathrm{~m} \Omega$ from the copper conductor losses. Within the pass-band there is a significant difference between the two impedances and their frequency dependence.

The parallel END case input impedance has a maximum real component near the center of the band falling to near zero at the band edges, while its imaginary component peaks at the lower band edge, falls to zero at the resonant frequency $f_{\mathrm{o}}$ and then goes negative with a negative peak near the top of the band.

The parallel MID case exhibits a real input impedance that is peaked near the band edges and has a minimum within the pass-band above the resonant frequency. For the majority of the pass-band the real value only varies by $\pm 15 \%$ about $46.3 \Omega$ for $65 \%$ of the bandwidth. The imaginary component of the parallel MID input impedance has a nearly frequency independent value for most of the pass-band which is negative (with a value of $-11.7 \Omega$ for these parameters).

The series connections both exhibit a much smaller impedance than the parallel version. Both real components are smoothly peaked near to the resonant frequency of 13.6 $\mathrm{MHz}$ with the MID case having nearly twice the real resistance as the END. Most attractive is that the imaginary part of the MID input impedance is very close to zero for the whole passband $\left(-0.2<X_{M I D}<0.2\right)$.

Conventionally the maximum power transfer theorem suggests that maximum power is provided from a source to a load when both have the same impedance. In such a case the efficiency is only $50 \%$ as half the power generated by the source is lost internally. Hence for high-efficiency power transfer, it is preferable to mismatch the source and load with the source impedance being as low possible. Thus, in order to maximize the power transfer efficiency for this system the parallel connection is preferred as this presents a much higher input impedance than the series variant. Power transfer only requires that a single frequency be transmitted but this introduces problems when reflections from the ends of finite lines are considered. Standing waves resulting from such reflections will modulate the smooth curves for impedance and reflection. 

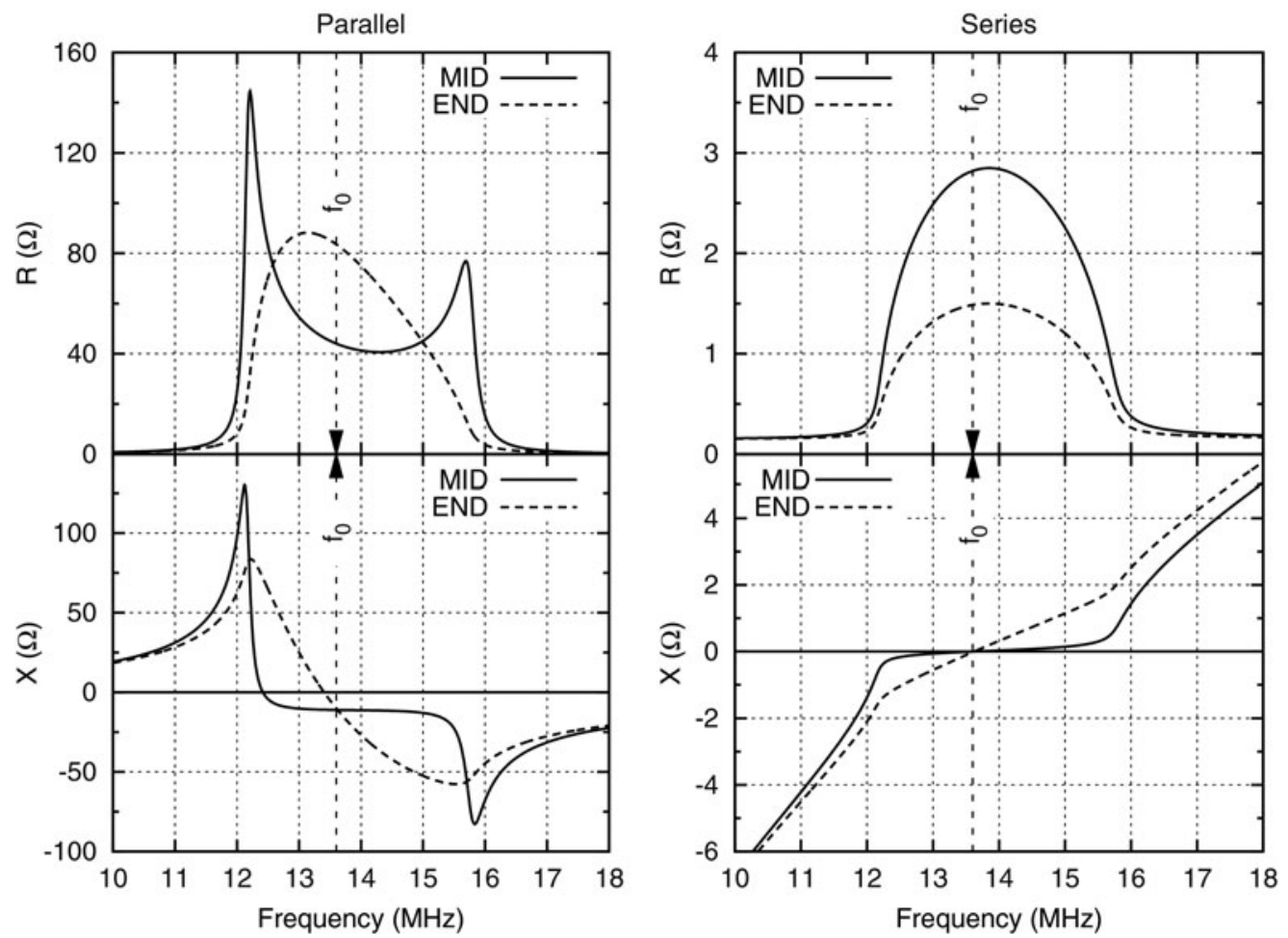

Fig. 3. Input impedances for sources connected in the two locations (END and MID) in both parallel and series configuration. Resonant frequency of $13.6 \mathrm{MHz}$ is indicated. Cell parameters in this case are $L=130 \mathrm{nH}, C=1.05 \mathrm{nF}, R=88 \mathrm{~m} \Omega, M_{1}=-16.5 \mathrm{nH}$, and $|k Q|=32$.

\section{C) Finite lines}

As alluded to at the end of Section IIB real lines will be finite so that standing waves are expected to impact the performance for most applications unless the line ends are terminated. In a power transfer system absorbing terminations for the MIW are quite undesirable as they will lead to large fixed losses and seriously degrade overall efficiency unless they are themselves the loads to which power is being provided. Hence, we confine ourselves to considering unterminated lines where the only losses are those intrinsic to the line (the resonator loss $R$ ), the source loss $R_{s}$ and one or more loads inductively coupled to the line. To perform finite line calculations we adopt the circuit modeling approach as has been employed previously [10] to compute currents in each cell of a line and each receiver coupled to it. In brief this method uses the impedance matrix $(\mathbf{Z})$ for the set of coupled cells and receiver which is numerically inverted for each frequency under consideration and then used to determine the currents $\mathbf{i}$ circulating in the cells of the device driven by the source(s) (V).

$$
\begin{gathered}
\mathbf{V}=\mathbf{Z i} \\
\mathbf{i}=\mathbf{Z}^{-1} \mathbf{V}
\end{gathered}
$$
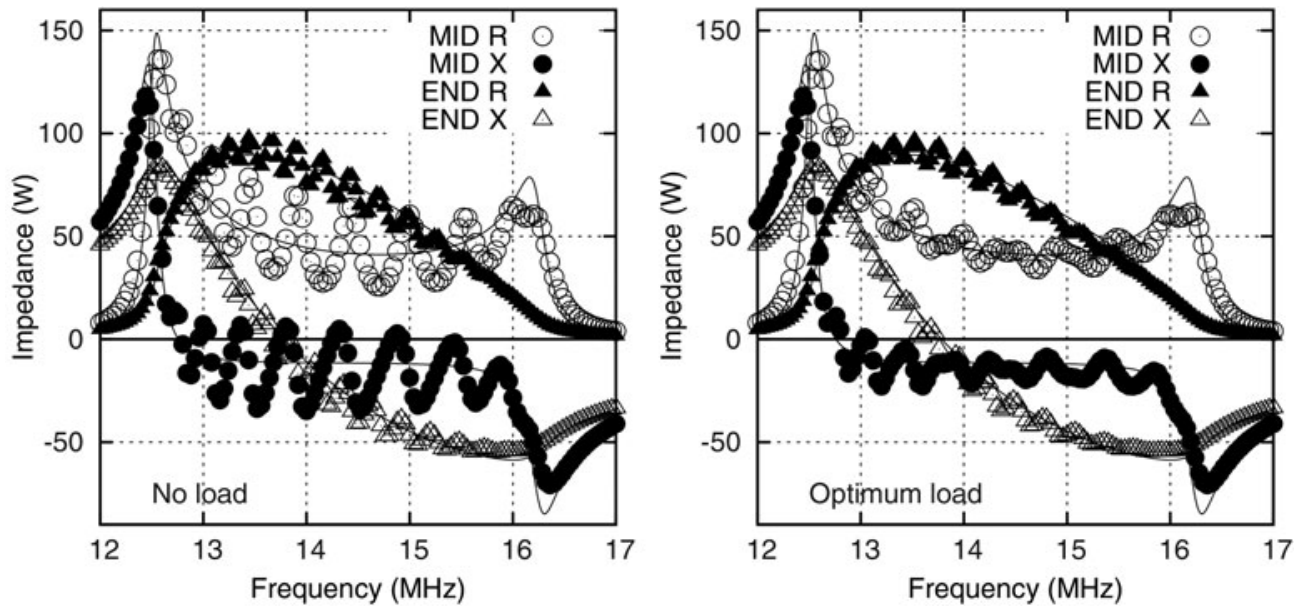

Fig. 4. (Left) parallel coupled input impedance for MIW line 21 cells long computed at the center (MID) and the ends (END) without a receiver and load. (Right) the same calculation but with a load present and strongly coupled to an end cell. Lines are infinite model calculations from Fig. 3. Circuit parameters are identical to those used for infinite model with $M_{2}=-0.60 \mathrm{nH}$ and $M_{3}=-0.96 \mathrm{nH}$. Receiver coupling is $57.0 \mathrm{nH}$ to the end cell and $-8.3 \mathrm{nH}$ to the next nearest. 
Circuit modeling uses the matrix inversion method described elsewhere [15] and in this case we include coupling terms to third-order (1st, 2nd, and 3rd neighbor mutual inductances: $M_{1}, M_{2}$, and $M_{3}$, repectively), are included for the cells forming the lines and for the receiver elements. To compute the input impedance the current drawn from a source is calculated for each frequency and compared with the potential generated across the input of the MIW. Output power is computed by adding a load to the receiver element which is otherwise identical to those forming the line. Loads are connected to a receiver inductively coupled to the five cells closest to it. The load impedance may be either series connected with the $L R C$ elements of the receiver resonator, or in parallel across its capacitance, as shown in the two receivers of Fig. 1.

\section{1) INPUT IMPEDANCE AND STANDING WAVES}

Figure 4 (left pane) shows the input impedance calculated for a finite 21 cell line excited either at its first cell (END parallel) or at cell number 11 (MID parallel) compared with the infinite line calculations from Fig. 3. Cell parameters are the same as those used previously with extra values computed using FastHenry [17] for the second and third nearest-neighbor couplings. To obtain these results the receiver is effectively removed by setting its coupling inductances to zero. In all source cases, the effects of standing waves are to modulate the input impedance components about the infinite model result. The amplitude of the standing waves is clearly greater for the MID case as would be expected (waves only have to travel half as far to the reflective ends of the structure for the MID as compared with END case). In general, for highpower transfer efficiency one would like to minimize the cell resistance in order to ensure cell losses are as insignificant as possible. The results of Fig. 4 (left) show that this would offer a penalty in terms of the standing waves influence.

The right pane of Fig. 4 shows the results for the input impedances when a load is coupled to the end of line (opposite to the end used for the END case). This receiver has a load of 5 $\Omega$ connected in series with its LRC components and is strongly coupled to the line (set at a distance of $3 \mathrm{~mm}$ above for FastHenry [17] calculations). The modulation depth of the standing waves is significantly reduced in this case as $70 \%$ of the power arriving at this end is now being absorbed by the load.

Two conclusions can be drawn from this result. Firstly, standing waves are not a serious impediment to power transfer as, when one has a well-coupled load they are suppressed removing the strong frequency variation in input impedance that they generate for the unloaded line. Secondly, the infinite model input impedances are in fact quite close to those for the finite case when the load is strongly coupled making these results useful in determining optimal source and receiver parameters.

\section{2) POWER TRANSFER EFFICIENCY}

With a single receiver located above one of the cells of the line, power is sent to that receiver's load by the source driving the line. Power efficiency is calculated including the source losses in $R_{s}$ using circuit modeling and matrix inversion as described elsewhere [10] to find the currents in each cell plus the receiver, then using them to obtain power at the load $\left(P_{L O A D}=\left|i_{R}\right|^{2} R_{L}\right)$, while power from the source is evaluated as the real part of the complex product of source voltage and current $\left(P_{\text {input }}=\mathfrak{k}\left(V_{S} i_{\text {input }}^{*}\right)\right)$. The maximum voltage $\left(V_{B}\right)$ that may be applied to a line is limited by the breakdown potential of the capacitor used to set the cell's resonant frequency.

Figure 5 shows the results of one such calculation for a line, consisting of 21 cells and 1 receiver. Each cell is the same as those used for the impedance calculations previously and all parameters are the same. A low impedance source of $1 \Omega$ is connected in parallel to the central cell (the 11th in the line) and the load resistance and receiver height allowed to vary. The load is connected in series with a receiver cell located at a height $z(1-20 \mathrm{~mm})$ above each of the cells of the line in turn. The receiver here is identical to the cells of the line which are the same $a=40 \mathrm{~mm}$ square loops as introduced earlier.

Three cases are considered here. Firstly driving the source at a fixed frequency $(13.6 \mathrm{MHz})$ and then seeking the combination of $R_{L}$ and height $z$ that give the largest efficiency. For this case we show the power output under these conditions along with the load resistance and the height that give the maximum efficiency. The second and third cases are obtained by allowing the drive frequency to vary over the entire MIW band and finding the combination of $R_{L}, z, f$ that give maximum efficiency ('opt' case) and maximum output power ('maxP' case)

Efficiency: Figure 5(a). Fixed frequency efficiency $\left(\eta_{13.6}\right)$ peaks at $77 \%$ in the degenerate case (receiver directly above the source at cell 11) to $1.9 \%$ for the 2 nd and 20 th cell locations. The even numbered receiver locations have much lower efficiency and power than the odd numbered positions. The origin of these oscillations is the generation of standing waves between the source and the cell coupled to the load from which waves are reflected. The resonant frequency has a wavelength of exactly four cells resulting in standing wave peaks every 2 cells. Turning to the maximum efficiency $\left(\eta_{\text {opt }}\right)$ one finds a higher degenerate peak of $93 \%$ and the lowest value at the ends of the line of $38 \%$. The peak arises in the region where the direct coupling between source and receiver adds to the power transferred via MIW to enhance the efficiency. At a distance of two cells the direct coupling becomes insignificant and all power is being delivered via the MIW line. Maximum output power efficiency $\left(\eta_{\max P}\right)$ is only $51 \%$ and drops to $24 \%$ at the ends of the line. It is notable that the maximum efficiency coincides with that for the fixed resonant frequency drive of $13.6 \mathrm{MHz}$ for the odd numbered cells where standing wave peaks are generated.

Output power: Looking at the output power (Fig. 5(b)), values for the fixed frequency vary between $3.5 \mathrm{~W}$ and 13 $\mathrm{mW}$ due to the standing wave interference, while the maximum efficiency and maximum power cases result in more consistent performance. The maximum efficiency case gives output powers ranging from $2.4 \mathrm{~W}\left(x_{r}=11\right)$ to $0.6 \mathrm{~W}$ at the ends. The majority of the degradation in both output power and efficiency arises from the attenuation of the MIW over the ten cells between source and the ends of the lines. Maximum output power peaks at $17.7 \mathrm{~W}$ dropping to $0.93 \mathrm{~W}$ at the ends. Once again the maximum efficiency, maximum output power cases and peak $13.6 \mathrm{MHz}$ values tend to converge away from the source cell.

Frequency: The frequencies corresponding to the three cases are shown in Fig. 5(c). Away from the central source cell these are close to, but generally above the resonance frequency varying between 13.6 and $15.5 \mathrm{MHz}$. 


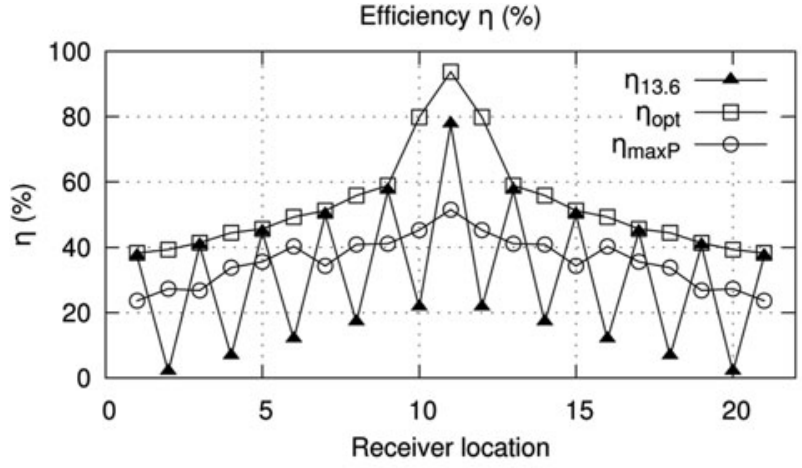

(a)

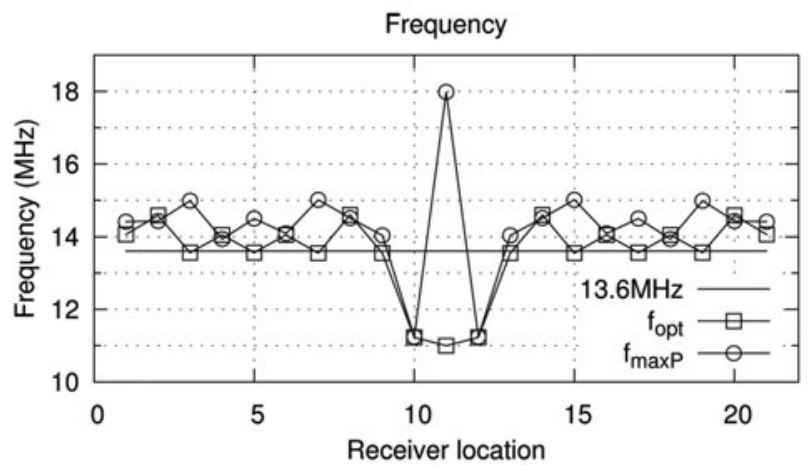

(c)

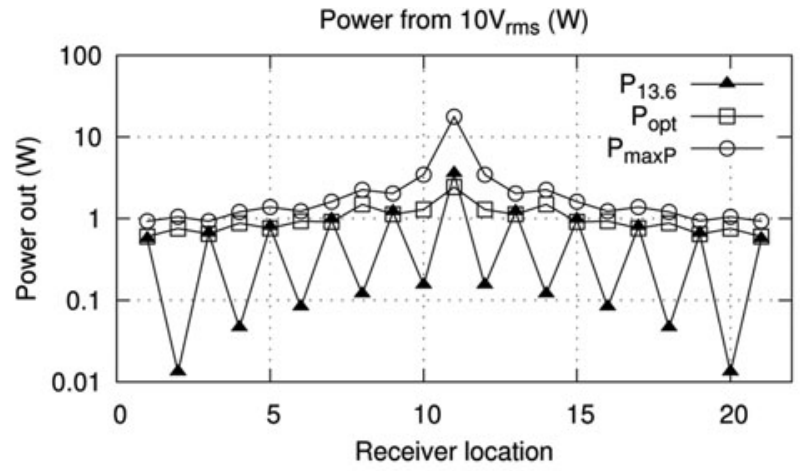

(b)

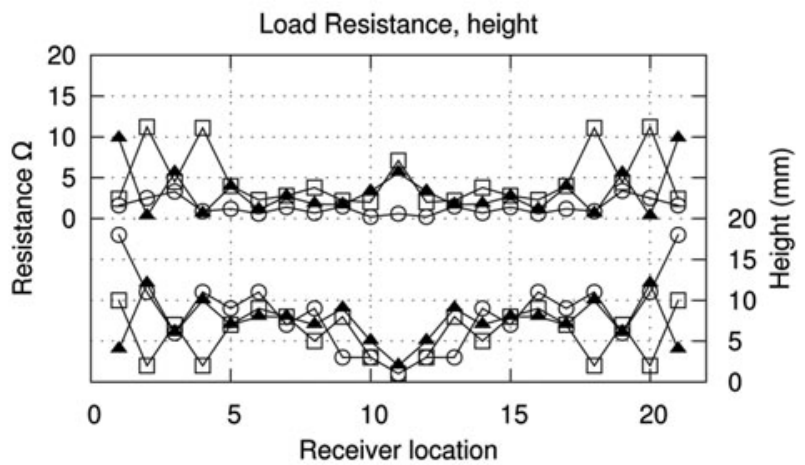

(d)

Fig. 5. Predicted performance parameters [efficiency (a), output power (b), operating frequency (c), load resistance and receiver height (d)] for a 21 cell line driven by a source connected to its central cell. Three cases are shown for properties if driven at a fixed frequency of $13.6 \mathrm{MHz}$, at the frequency giving maximum efficiency $\left(f_{\mathrm{o}} p t\right)$ and at that frequency that generates maximum output power.

Load Resistance and height: Finally the optimal load resistances and receiver heights have been evaluated as shown in Fig. 5(d). Standing waves near the line ends result in large variation in these parameters but away from them the optimum load resistances lie between 1.5 and $4 \Omega$, while the optimal height is close to $7 \mathrm{~mm}$. It is useful, at this point, to make some observations based on the above data. Firstly at least for this type of capacitor loaded resonator, the overall length in cells should be short to minimize attenuation losses. If a fixed drive frequency is required (as is likely given the licensing restrictions on RF systems), then the resonant frequency for the cells of the line should be chosen lower

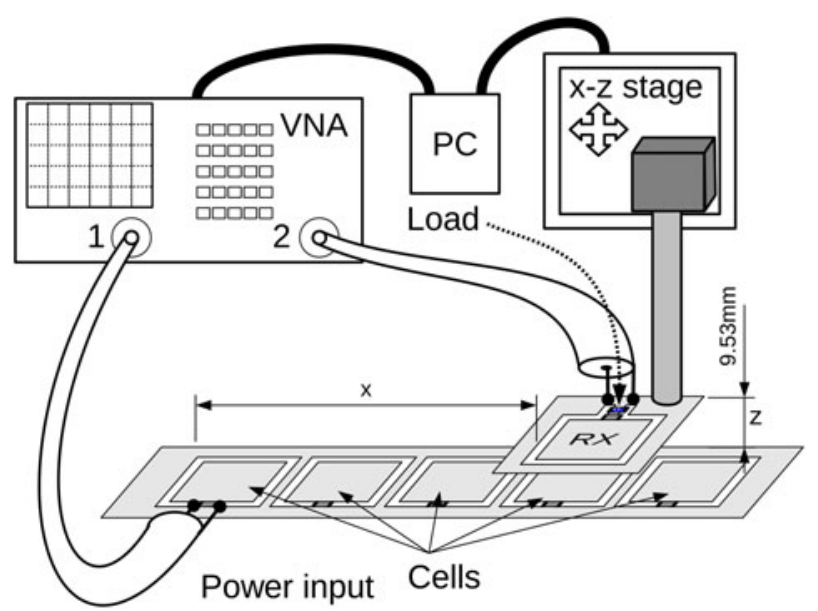

Fig. 6. Experimental apparatus for measurements of power transfer. than the drive frequency for best performance. For best performance the drive frequency should be allowed to vary between 13.6 and $15 \mathrm{MHz}$. In fact, it is possible that only two drive frequencies (13.6 and $14.5 \mathrm{MHz}$ ) would be required to give near optimal performance at all cells.

\section{EXPERIMENTAL VERIFICATION}

To confirm the predictions of the circuit-based modeling described in the previous section a simple experimental verification has been conducted. The 21 cell line made from $a=$ $40 \mathrm{~mm}$ cells spaced $g=0.2 \mathrm{~mm}$ apart would be nearly 850 $\mathrm{mm}$ long and was impractical to fabricate for a test of this kind. We instead constructed a smaller five cell line whose total length is $201 \mathrm{~mm}$. Tracks were laid out $w=1 \mathrm{~mm}$ wide and fabricated by PWCircuits Ltd. [18] on an FR4 substrate using photo-lithography. Each cell was a square single turn loop and had a maximum outer dimension of $40 \times 40$ $\mathrm{mm}$ with a $0.2 \mathrm{~mm}$ gap between cells. Surface mount ceramic multilayer capacitors ( $1 \mathrm{nF} 603$ size, Co G dielectric Kemet Ltd.) were soldered across gaps in the tracks to form a resonator. The power receiver is a resonator of identical dimensions to the MIW cells but has an extra gap across which the series load of $3.3 \Omega$ is connected.

Initially measurements of individual resonators loaded with $1 \mathrm{nF}$ capacitors gave a resonant frequency of 13.75 $\mathrm{MHz}$ with a $Q$ factor of 37 . Hence, the real inductance of the individual resonators was $134 \mathrm{nH}$. Using a pair of cells the effective first-order coupling between them was determined from the split of their resonances giving a value for 
M1 of $-16.33 \mathrm{nH}$, very close to the predicted values of $L=$ $130 \mathrm{nH}$ and $\mathrm{M}_{1}=-16.5 \mathrm{nH}$ from FastHenry [17]. The $Q$ factor implies a cell equivalent resistance of $307 \mathrm{~m} \Omega$ which is rather higher than the $88 \mathrm{~m} \Omega$ copper loss value used in the simulations reflecting the significant extra losses from solder (typically $100 \mathrm{~m} \Omega$ per joint), the ceramic capacitors equivalent series resistance $(11 \mathrm{~mW}$ from the manufacturers datasheets) and possibly some radiation loss. This larger resistances inevitably increase the losses associated with MIW propagation for this system but as we are only using five cells it should be tolerable. The figure of merit for this structure $|k Q|=9.1$ in contrast to the figure of 32 expected for the copper loss alone.

The experimental apparatus is shown in Fig. 6. Measurements were made using a vector network analyser (VNA) to provide the source (connecting directly across the central cell's capacitor) and to measure the received signals connecting across the load resistance. Care must be taken when analysing data using the VNA $\left(Z_{\mathrm{o}}=50 \Omega\right)$ to adjust for the difference between the VNA impedance and the actual load resistance. In order to convert the measured forward scattering parameters to power transfer efficiency (= power to load/power into line) one must combine the forward and reverse scattering parameters to find the effective efficiency from a simple source as shown in equation (7).

$$
\eta_{\text {power }}=\frac{S_{21}^{2}\left(1+Z_{0} / R_{L}\right)}{1-S_{11}^{2}},
$$

where $Z_{\mathrm{o}}$ is the characteristic input impedance of the VNA (50 $\Omega \mathrm{W})$ and $R_{L}$ is the load resistance across which the output is measured. Hence for this $3.3 \Omega$ load the power efficiency is more than 16.1 times the measured value for the forward scattering parameter. This efficiency does not however include any source losses as the data has effectively been corrected
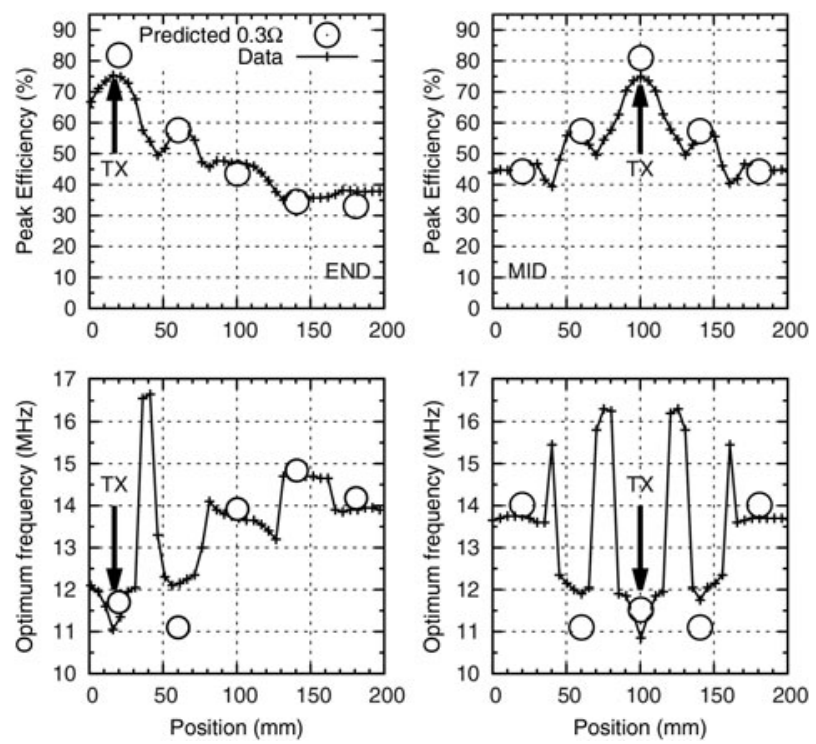

Fig. 7. (top left) Measured peak power transfer efficiency for a line with source driving one end (crosses) compared with predicted values for receiver aligned with each cell in turn (circles). Here the calculations are made with a cell resistance of $0.3 \Omega$ matching the experimental values derived from measured $Q$ factors. (bottom left) Optimum frequency for maximum efficiency with END drive. (top right) Peak efficiency for MID drive. (bottom right) Optimum frequency for this case. for them by this process. To compare with a model calculation one must set the source impedance to a very low value. In a realistic implementation of course, there will be some source losses which reduce the overall system efficiency below these values.

The receiver is swept along the line in $5 \mathrm{~mm}$ steps for heights above the line in $1 \mathrm{~mm}$ increments with the closest approach between the copper layers of the line and receiver being $1.6 \mathrm{~mm}$. Maximum separation between line and receiver was $26.6 \mathrm{~mm}$. Two source connections were evaluated - one in the MID position (cell 3 ) and one at the END location (cell 1). Both cases were modeled using the same circuit method previously described for comparison with $|k Q|=9.1$ as determined from the discrete resonator performance. Figure 7 shows the peak efficiency for each longitudinal position of the receiver along with the frequency for this maximum performance for the two source configurations. We also plot discrete points for the computed model power transfer efficiency corresponding to the five locations where the receiver is aligned to one of the MIW cells. Maximum efficiency in both cases occurs when the receiver is aligned (location TX in the figure) with the transmitter and is $75 \%$ for both END and MID cases. This drops quickly and by the time the receiver is two cells away from the source the efficiency has dropped to around $45 \%$. The rapid drop here is attributable to a combination of the rapid falloff of the direct coupling between source cell and receiver adding to the MIWs' attenuation. After this the dominant power transfer mechanism is via MIWs. The frequencies at which these maximal efficiencies are obtained are also shown. There are large variations in the optimal frequency near the transmitter but these converge to a value just above the resonant frequency away from the source. The calculated power transfer efficiency matches most of the locations quite well but the TX point generally has a higher predicted efficiency than the measured value. Inspecting the height for which these maximum efficiencies are obtained at the TX point is always the minimum value of $1.6 \mathrm{~mm}$. At this point some capacitive coupling between the TX cell and the receiver is to be expected which generally has opposite sign to the magnetic coupling for this configuration and hence acts to reduce the actual power transferred.

At more than two cells horizontal displacement from TX MIW dominate the power transfer and the strong MIW attenuation present in our test structure becomes the main limitation on overall efficiency.

\section{DESIGN METHDDOLDGY AND DPTIMIZATION}

By understanding the MIW properties of the line one may rapidly find the potential performance of any relay coil/ MIW device. For a series connected source, driving one end of a semi-infinite line, the input impedance at resonance is very close to $\omega_{0} M$ and approximately double to this for mid-line sources. This is also true for finite lines, when they are well terminated by an absorbing load (possibly that to which power is being supplied). Attenuation on the line is given by equation (1) and longer the line the greater the loss for power transfer to the ends given by $1-e^{-\alpha d}$. In order to perfectly match the line the load must introduce an effective impedance to the line equal to the series input impedance. 

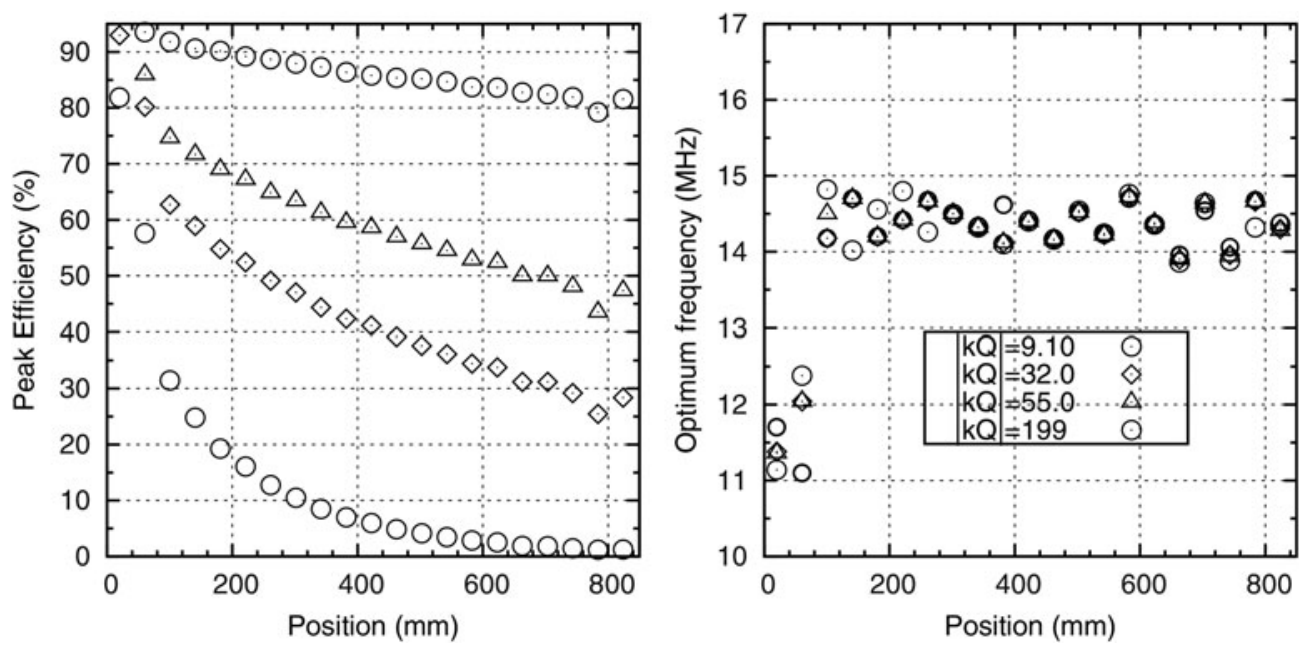

Fig. 8. Predicted performance for a 21 cell line with $|k Q|=9.1,32,55$, and 199. (left) Power transfer efficiency for an END connected source and (right) optimum frequency.

This in turn determines the height or a receiver terminal and its load. These design rules can be expressed in the simple form of equation (8), where $n_{D}$ is 1 for a semi-infinite line and 2 for an infinite case and the approximation on attenuation holds well for $k Q>5$. The expressions assume perfect termination at the resonant frequency by a load whose impedance is equal to the input impedance.

$$
\begin{aligned}
Z_{\text {in }}\left(\omega_{0}\right) & \approx n_{D} \omega_{0} M \\
\alpha d & \approx f r a c 1 k Q, \\
\eta_{\max } & \leq 1-e^{-\alpha d} \approx 1-e^{11 / k Q} .
\end{aligned}
$$

It is clear from the rather low values of the experimental efficiency that the higher than anticipated effective cell resistance and reduction of the figure of merit to 9.1 from 32 has resulted in too much loss. Future design for metamaterial power transfer could counter this by several strategies:

Multiple turn inductors: As the product $k Q$ controls the magnitude of attenuation for MIW increasing $Q$ by raising the resonator inductance seems an obvious choice. In isolation $L$ may be increased by a very large factor using spiral structures with increasing numbers of turns. While this raises the resistance linearly it raises $L$ in a super linear fashion so that their ratio controlling $Q(=\omega L / R)$ can be significantly enhanced. If one is winding coils on cylindrical cores this is very effective but leads to a rather thick and complex structure in terms of manufacturing. $Q$ factors as high as 800 have been reported for such coils [19]. For planar PCB inductors multiple turns can be achieved using spiral patterns but care must be taken as, should the inner dimension of the spiral become too small, the value of $\mathrm{k}$ will decrease. Using the wheeler method for square coils, a three turn spiral with 1 $\mathrm{mm}$ track widths and inter track gaps of $0.2 \mathrm{~mm}$ made from $35 \mu \mathrm{m}$ thick copper would offer $Q$ in excess of 180 but will reduce $k$ to -0.20 giving $|k Q|=36$.

Self-resonant structures: In our test structure the resonators' resonances were set to $13.56 \mathrm{MHz}$ by adding a low loss surface mount capacitor to each cell. This added series resistance and degraded the overall $Q$ factor. Using resonator structures that do not require this lumped element offers potential for very much higher $Q$ values and longer range power transfer structures. Planar spirals, broadside coupled split rings, multi-layer spirals [16], and others all offer higher Q possibilities but are difficult to design for frequencies as low as 13.6 $\mathrm{MHz}$. Wound self-resonant coils can offer $Q$ factors in excess of 1400 but present manufacturing penalties [19].

Enhanced coupling: Our test structure is a purely singlelayer planar design but this imposes strong limitations on the maximum possible coupling and the value we achieve of $k=-0.24$ is close to the realistic limit for symmetric cells of this type. The coupling can however be much larger if structures use more than one later and the cells of each layer are overlapped [20]. Alternately quasi-axial structures using cells each of which overlays the next by half its width also give good performance with coupling reported at $k=0.675$ [21] although these have only been demonstrated using cells with very high aspect ratios (very long in the line direction). Using one or more of these approaches it is thus possible to significantly improve on the performance of our test structure. Using the circuit modeling method we have investigated how much efficiency could be improved as the $k Q$ value increases. Literature values for magneto-inductive cables for instance [21], give $Q$ of 48 and $k=0.675$ to a total of 32 . To explore the potential we calculate the power transfer to our single turn terminal for a variety of cell resistances and $k Q$ values and the results are shown in Fig. 8.

For a 21 cell line $(844.2 \mathrm{~mm}$ long) our original cells $(|k Q|=$ 9.1) would offer $<_{4} \%$ power transfer efficiency end to end while cells with $|k Q|=32$ could achieve in excess of $30 \%$ with better than $60 \%$ for lines of $400 \mathrm{~mm}$ or less. If one were to use the self resonant cells of reference [21] $|k Q|=$ 55 is possible offering $50 \%$ for 21 cell lines. Using selfresonant coils taken from the literature [19] one may achieve $|k Q|=199$ with $80 \%$ efficiency for the 21 cell line and better than $90 \%$ over 11 cells. It is important to note here that the frequencies for optimal power transfer shown for all these cases (Fig. 8 left) do not significantly vary as a function of the $|k Q|$ value being only due to the effects of standing waves rather than any spectral variation of the attenuation within the passband. Hence optimization for power transport need only consider maximizing the $|k Q|$ product. 


\section{CONCLUSIONS}

This study has investigated the potential to transfer power efficiently between source and load via MIWs traveling on a $1 \mathrm{D}$ metamaterial waveguide. We have found that it is indeed possible to transfer useful power via MIWs with the breakdown of the resonating capacitors being the principal limitation. From this understanding of the nature of power flow along such a line we have developed simple methods to determine performance and obtain optimal design using very simple analytical formulae. Although experimental efficiency of only $40 \%$ was demonstrated potential exists for much higher performance if our resonators' design can be improved. Power transfer is maximized when the product of the dimensionless coupling $k(=2 L / M)$ and the cells resonant $Q$ factor is maximized. Standing waves on low loss lines do not significantly limit power transfer but do result in a variation in the frequency for optimal efficiency. For $1 \mathrm{D}$ lines such as those explored here it is possible to obtain reasonable power transfer using two drive frequencies both of which are somewhat higher than the cell's resonance. Driving the MIW cells directly from a source is best achieved with a low impedance source connected in parallel and maximum power is extracted from the receiver if the load is placed as a series components in its resonator. The matching impedance in the experimental case was $3 \Omega$ but use of cells with a larger mutual inductance would (as shown in equation (4)) offer a larger optimum load value. It is possible that better performance still could be obtained if the receiver were itself formed using a higher $Q$ structure with more turns. Finally, it is useful to point out that MIW are present both in $1 \mathrm{D}$ and $2 \mathrm{D}$ structures offering the potential for power transfer surfaces covering relatively large areas if resonator design can be optimized. Such $2 \mathrm{D}$ structures exhibit larger bandwidths and lower loss than $1 \mathrm{D}$ waveguides but still offer the near field power and data coupling to simple receivers.

\section{ACKNDWLEDGEMENTS}

I gratefully acknowledge the support of Isis Innovations Limited in this work.

\section{REFERENCES}

[1] Tesla, N.: The transmission of electric energy without wires. Electr. World Eng., 5 (1904), 162.

[2] Kurs, A.; Karalis, A.; Moffatt, R.; Joannopoulos, J.D.; Fisher, P.; Soljavcić, M.: Wireless power transfer via strongly coupled magnetic resonances. Science, 317 (5834) (2007), 83-86.

[3] van Wageningen, D.; Staring, T.: The Qi wireless power standard. in 14th Int. Power Electronics and Motion Control Conf. (EPE/PEMC), 2010, (2010), S15-25.

[4] Urzhumov, Y.; Smith, D.R.: Metamaterial-enhanced coupling between magnetic dipoles for efficient wireless power transfer. Phys. Rev. B, 83 (20) (2011), 205114.

[5] Stevens, C.J.: Power transfer via metamaterials. Compt. Mater. Con., 33 (1) (2013), 1-18

[6] Wang, B.; Yerazunis, W.; Teo, K.H.: Wireless power transfer: metamaterials and array of coupled resonators. Proc. IEEE, 101 (2013), 1359-1368.
[7] Lee, C.K.; Zhong, W.; Hui, S.Y.R.: Effects of magnetic coupling of nonadjacent resonators on wireless power domino-resonator systems. IEEE Trans. Power Electron., 27 (2012), 1905-1916.

[8] Narusue, Y.; Kawahara, Y.; Asami, T.: Impedance matching method for any-hop straight wireless power transmission using magnetic resonance, in 2013 IEEE Radio and Wireless Symp. (RWS), (2013), 193-195.

[9] Mori, K.; Lim, H.; Iguchi, S.; Ishida, K.; Takamiya, M.; Sakurai, T.: Positioning-free resonant wireless power transmission sheet with Staggered Repeater Coil Array (SRCA). IEEE Antennas Wireless Propag. Lett., 11 (2012), 1710-1713.

[10] Stevens, C.J.; Chan, C.W.; Stamatis, K.; Edwards, D.J.: Magnetic metamaterials as 1-D data transfer channels: an application for magneto-inductive waves. IEEE Trans. Microw. Theory Tech., , 58 (5) (2010), 1248-1256.

[11] Shamonina, E.; Kalinin, V.A.; Ringhofer, K.H.; Solymar, L.: Magnetoinductive waves in one, two, and three dimensions. J. Appl. Phys., 92 (10) (2002), 6252-6261.

[12] Shamonina, E.; Kalinin, V.A.; Ringhofer, K.H.; Solymar, L.: Magneto-inductive waveguide. Electron. Lett., 38 (8) (2002), 371-373.

[13] Chan, C.; Stevens, C.: Two-dimensional magneto-inductive wave data structures. in Proc. of the 5th European Conf. on Antennas and Propagation (EUCAP), (2011), 1071-1075.

[14] Wiltshire, M.; Hajnal, J.; Pendry, J.; Edwards, D.; Stevens, C.: Metamaterial endoscope for magnetic field transfer: near field imaging with magnetic wires. Opt. Express, 11 (2003), 709-715.

[15] Solymar, L.: Waves in Metamaterials. Oxford University Press, Oxford, 2009.

[16] Hao, T.; Stevens, C.; Edwards, D.: Optimisation of metamaterials by Q factor. Electron. Lett., 41 (11) (2005), 653-654.

[17] Kamon, M.; Tsuk, M.J.; White, J.K.: FASTHENRY: a multipoleaccelerated 3-D inductance extraction program. IEEE Trans. Microw. Theory Tech., , 42 (9) (1994), 1750-1758.

[18] PW Circuits Ltd.: Premier Works, Canal Street, South Wigston, Leicester, England LE18 $4 \mathrm{PN}$.

[19] Breitkreutz, B.; Henke, H.: Calculation of self-resonant spiral coils for wireless power transfer systems with a transmission line approach. IEEE Trans. Magn., 49 (9) (2013), 5035-5042.

[20] Radkovskaya, A.; Sydoruk, O.; Shamonin, M.; Stevens, C.J.; Faulkner, G.; Edwards, D.J.; Shamonina, E.; Solymar, L.: Transmission properties of two shifted magnetoinductive waveguides. Microw. Opt. Technol. Lett., 49 (5) (2007), 1054-1058.

[21] Syms, R.R.A.; Solymar, L.; Young, I.R.; Floume, T.: Thin-film magneto-inductive cables. J. Phys. D: Appl. Phys., 43 (5) (2010), 055102.

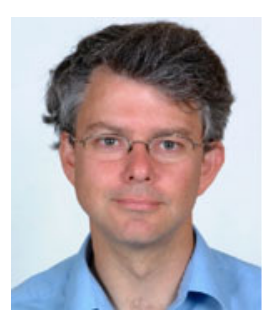

Christopher J. Stevens read physics at the University of Oxford, UK, graduating in 1990 with first class honours and prizes for practical physics and received his D. Phil. degree in condensed matter physics in 1994 following a 3 -year doctoral program at the same university. Subsequently he worked in Uiversita Degli Studidi Lecce after which he held a Royal Academy of Engineering fellowship at St. Hugh's College, Oxford. He now holds an engineering faculty position at the University of Oxford and is a Fellow of St. Hugh's College, Oxford. His current research activities include ultrawideband communications, metamaterials, ultrafast nanoelectronics, and high-speed electromagnetics. 